\title{
Medición de la satisfacción de los usuarios directos del Departamento Programas sobre el Ambiente del Servicio de Salud Aconcagua
}

\author{
FANCY MELENDEZ(1), OSCAR ARTEAGA(2), ENRIQUE HERNÁNDEZ (3)
}

\section{RFSUMEN}

El objetivo de este estudio fue evaluar el grado de satisfacción de los usuarios directos del Departamento de Programas sobre el Ambiente del Servicio de Salud Aconcagua respecto a la atención recibida. Para ello se aplicó una encuesta a una muestra representativa de 307 usuarios atendidos entre los meses de octubre del 2002 y febrero del 2003. El instrumento utilizado fue diseñado especialmente para este estudio. Las dimensiones consideradas fueron accesibilidad, infraestructura, relaciones interpersonales, calidad técnica y satisfacción global y las variables sexo, edad, nivel educacional, tipo de trámite y oficina de atención. Entre los hallazgos obtenidos, destacan el alto porcentaje de satisfacción con la atención brindada $(73,61 \%$ ) y que la variable mejor evaluada fue el trato brindado por los funcionarios (sobre $90 \%$ en todos los casos). También destaca que la variable que obtuvo más baja calificación fue la capacidad resolutiva de las denuncias con sólo un $18,6 \%$ de conformidad con la solución entregada. El estudio de asociaciones permitió concluir que las variables que más impactan la evaluación de la satisfacción global son la claridad de la información y el tiempo de tramitación de las solicitudes. Otros hallazgos indican que los usuarios que realizaron trámites más complejos y denuncias otorgaron una calificación más baja al tiempo de tramitación $(p<0,05)$, y que ambos tipos de trámites fueron realizados con más frecuencia por mujeres $(p<0,05)$, quienes -a su vez-tienen un nivel educacional más bajo $(p<0,05)$ y evaluaron más mal la claridad de la información $(p<0.1)$.

Palabras clave: Satisfacción ustuaria, Ambiente.

\section{ABSTRACT}

\section{MEASUREMENT OF THE SATISFACIION OF THE DIRECT USERS OF THE DEPARTMENT PROGRAMS ON THE ATMOSPHERE OF THE SERVICE OF ACONCAGUA HEALTH}

The objective of this study was to assess the satisfaction degree of direct users of Aconcagua Health Service's Department of Environmental Programs. For this purpose, a survey was applied to a representative sample of 307 attended users between the months of October 2002 and February 2003. The applied instrument was specially designed for this study. Accessibility, infrastructure, interpersonal relationships, technical quality and global satisfaction were the dimensions considered. Sex, age, educational level, kind of procedire, and attention office variables were also considered. The high percentage of satisfaction due the rendered attention (73.6I\%) and the treatment offered by the officers as the best evaluated variable (over $90 \%$ in all the cases), were some of the findings of this study. The

\footnotetext{
(1) Departamento Programas sobre el Ambiente Servicio de Salud Aconcagua. Chile. dirambiente@ssaconcagua.cl

(2) Escuela de Salud Pública. Universidad de Chile.

${ }^{(3)}$ Escuela de Salud Pública. Universidad de Chile.
} 
low rating of the resolving capacity for the complaints, with only a $18.6 \%$ of conformity with the solution, was also outstanding. The association analysis allowed to conclude that clarity of information and time of carrying out application are the most influential variables to the global satisfaction evaluation. Others findings were that users who perform more complex procedures and complaints gave a lower qualification to carrying out time $(p<0.05)$, both procedures were carried through be women $(p<0.05)$, who have a lower educational level $(p<0.05)$ and gave a worse evaluation to clarity of information $(p<0.1)$.

Key words: User satisfaction, Environment.

\section{INTRODUCCIÓN}

Uno de los ejes centrales de los procesos de reforma y de modernización del Estado en Chile y en otros países, es la calidad de la atención. Se reconoce que la satisfacción usuaria, es decir el grado de conformidad con el servicio recibido, es una dimensión que permite evaluar dicha calidad. La medición de la satisfacción usuaria tiene por propósito determinar las percepciones y actitudes del usuario. La subjetividad se transforma así en información relevante para los prestadores de servicios. De este modo, el nivel de satisfacción alcanzado no sólo depende de la calidad técnica de los servicios que se prestan, sino también de las expectativas del cliente. Consecuente con ello, conocer la opinión de los usuarios respecto a los servicios prestados debiera ser una meta ineludible de toda organización, ya que proporciona información estratégica para orientar su gestión a las necesidades sentidas de los usuarios ${ }^{1-4}$.

La modalidad de atención que se presta en salud ambiental ha sido muy poco estudiada en el país. Esto determina que la información generada en esta investigación no sólo sea valiosa para mejorar la gestión ambiental en el Servicio de Salud Aconcagua, sino eventualmente en otros servicios de salud. Se advierte que ella tiene potencial para contribuir a que el área de Salud Ambiental pueda asumir en mejor forma las funciones de salud pública que le son inherentes, así como los objetivos sanitarios fijados por el Ministerio de Salud 5 .

Entre los diversos servicios que prestan los Departamentos Programas sobre el Ambiente (DPA), descritos por Ordoñez ${ }^{6}$ y Urrutia ${ }^{7}$, destacan aquéllos que se otorgan a los usuarios directos, es decir a las personas que concurren a las oficinas que ha dispuesto el sistema de salud para resolver los reclamos ambientales y las solicitudes de permisos sanitarios.

Al plantear el estudio se esperaba que los prestadores pudieran identificar los problemas de la atención que afectan la satisfacción de los usuarios directos en los diferentes componentes de la atención, de manera de implementar acciones específicas que condujeran a mejorar los procedimientos aplicados en las oficinas de atención al usuario. Por otra parte, se esperaba contribuir a incorporar en la cultura organizacional de DPA la satisfacción usuaria al evaluar la calidad de las prestaciones, aspecto que hasta ahora no se había considerado dentro de sus lineamientos estratégicos.

El presente artículo forma parte de una investigación más amplia que combinó métodos cuantitativos y cualitativos. En esta comunicación sólo se presentan los hallazgos del componente cuantitativo.

\section{MATERIAL Y MÉTODO}

La metodología del presente estudio corresponde a un diseño transversal que usó una encuesta especialmente elaborada para esta investigación. En forma previa a la elaboración de la encuesta, se realizó un estudio cualitativo que permitió obtener información sobre los aspectos que interesaban a los usuarios. Esta información, además de antecedentes de otras investigaciones ${ }^{8-11}$, permitió elaborar el cuestionario. La encuesta consideró 37 preguntas cerradas para evaluar las dimensiones de accesibilidad, infraestructura, relaciones interpersonales, calidad técnica y satisfacción global y 5 preguntas de validación, dos de ellas cerradas y tres abiertas. El instrumento fue previamente probado y se le realizó análisis de confiabilidad mediante cl test de Kappa.

El universo a estudiar fueron los usuarios que tramitaron permisos sanitarios y denuncias en el período de un año. El tamaño de la muestra se de. 
terminó mediante el procedimiento de muestreo bietápico estratificado con afijación proporcional. Así la muestra quedó constituida por 307 usuarios. La muestra se extrajo de los usuarios que tramitaron solicitudes en el Departamento Programas sobre el Ambiente del Servicio de Salud Aconcagua durante los meses de octubre a diciembre del año 2002 y enero y febrero del año 2003

Previo consentimiento verbal, la encuesta se aplicó mediante entrevista cara a cara a cada usuario en su hogar, en el lugar de trabajo o en el establecimiento sujeto al permiso sanitario, una vez que éste había obtenido respuesta a la solicitud.

Las variables que median las dimensiones antes mencionadas se consideraron variables independientes, al igual que edad, sexo, nivel de educación, oficina de atención y tipo de trámite realizado. Se consideró como variable dependiente la evaluación de la satisfacción global.

Cada una de las variables fueron analizadas mediante distribución de frecuencias. Además se estudiaron asociaciones entre las variables independientes mediante test de $\chi^{2}$ y Fischer. Ello implicó dicotomizarlas de acuerdo a criterios de agrupamiento que fueron definidos, previo estudio de la distribución de frecuencias de cada una de ellas.

Finalmente, para intentar determinar las variables que más influyen en la satisfacción global de los usuarios, se estudió la asociación e impacto entre la variable dependiente satisfacción global y cada uno de las variables independientes. Para ello la variable satisfacción global fue agrupada en nota 6-7, lo que fue considerado como bueno a muy bueno, y 1-5, considerado como regular a muy malo. Esta sección del análisis consideró Intervalos de Confianza (I.C. 95\%), Riesgo Atribuible Porcentual (RAP\%) y Odds Rattio (OR).

Para todas las pruebas realizadas en el análisis estadístico se emplearon los programas computacionales Epilnfo 6.0 y STATA 6.0.

Las respuestas de las preguntas abiertas fueron codificadas de acuerdo a la dimensión a la que hicieran referencia y en el caso que aparecieron subfactores no considerados, se clasificaron y crearon códigos para ellas. Se analizó la consistencia de las respuestas de las preguntas de validación. con las entregadas por los usuarios en las preguntas que medían las diferentes dimensiones consideradas en el estudio.

\section{RESULTADOS}

Respecto a la caracterización de los usuarios, se observa que se distribuyen en forma similar entre hombres y mujeres (Tabla 1), el promedio de edad es de 42 años y sobre el $62,2 \%$ tiene nivel educacional igual o inferior al medio. Las tramitaciones más frecuentes realizadas (Tabla 2) corresponden a las definidas como poco complejas $(35,5 \%)$, las que incluyen locales del tipo almacenes, venta ambulante de alimentos no perecibles, licencias de operación de calderas y equipos de rayos $X$, entre otras y en segundo lugar las denuncias $(22,8 \%)$.

A continuación se presenta el resultado del grado de satisfacción para cada una de las dimensiones consideradas en el estudio.

La dimensión accesibilidad consideró las variables ubicación geográfica de las oficinas, horario de atención, tiempo de espera para ser atendido, tiempo de respuesta a la solicitud y costo de la atención. Las variables que mostraron más baja satisfacción (Tabla 3) fueron el horario de atención con un $63,8 \%$ calificado como bueno y muy bueno y el tiempo de tramitación de las solicitudes y el costo de la atención con un $62,8 \%$ y $46,8 \%$ calificado como adecuado, respectivamente.

Respecto a esta dimensión, para el $75 \%$ de los usuarios el tiempo de tramitación debería ser de no más de 8 días y el $71,4 \%$ desearía haber esperado hasta 10 minutos para ser atendido. El estudio permitió conocer támbién que el tiempo real de demora en la entrega del documento a los usuarios encuestados fue en promedio de 21 días, la moda de 7 días y los percentiles 50 y 75 de 13 y 26 días, respectivamente. Los tiempos promedios más largos de tramitación corresponden a las denuncias (30,5 días) y a los trámites más complejos (26,9 días). Se encontró una asociación estadísticamente significativa entre el tiempo de tramitación real y la evaluación de la demora en que hicieron los usuarios $(p<0,001)$, lo que significa que los trámites que efectivamente demoraron más tiempo, fueron los más mal evaluados.

La dimensión infraestructura consideró las variables espacio, limpieza y existencia de información escrita en las oficinas. Se observa que el espacio físico fue la variable más mal evaluada por los usuarios (Tabla 4), con sólo un $56,09 \%$ calificado como bueno a muy bueno, en comparación con el 
Tabla 1. Descripción porcentual de los 307 usuarios de las oficinas de atención usuaria del Departamento Programas sobre el Ambiente del Servicio de Salud Aconcagua, según sexo, nivel educacional y edad

\begin{tabular}{lrr}
\hline Variables & Frecuencia & $\%$ \\
\hline Sexo: & & \\
Masculino & 142 & 46,3 \\
Femenino & 165 & 53,7 \\
Nivel educacional: & & \\
Analfabeto & 2 & 0,7 \\
Básico & 59 & 19,2 \\
Medio & 130 & 42,3 \\
Técnico o Universitario incompleto & 26 & 8,5 \\
Técnico o Universitario completo & 90 & 29,3 \\
Edad segĺn rango: & & \\
18-29 & 53 & 17,3 \\
$30-41$ & 95 & 30,9 \\
$42-53$ & 103 & 33,6 \\
$54-65$ & 41 & 13,4 \\
$66-77$ & 15 & 4,9 \\
Total & 307 & 100,0 \\
\hline
\end{tabular}

Tabla 2. Distribución porcentual del tipo de trámite realizado por los usuarios atendidos en el Departamento Programas sobre el Ambiente del Servicio de Salud Aconcagua

\begin{tabular}{lrr}
\hline Tipo de trámite & Frecuencia & $\%$ \\
\hline Autorización, informe o certificado: & & \\
Alimento & 199 & 64,8 \\
Salud Ocupacional & 31 & 10,1 \\
Denuncia & 70 & 22,8 \\
Otro & 7 & 2,3 \\
Total & 307 & 100 \\
Grupo & & \\
Muy complejo & 28 & 9,12 \\
Complejo & 33 & 10,75 \\
Medianamente complejo & 67 & 21,82 \\
Poco complejo & 109 & 35,50 \\
Denuncias & 70 & 22,80 \\
Total & 307 & 100 \\
\hline
\end{tabular}

$94,0 \%$ y el $80,88 \%$ con que califica en esta categoría a la limpieza e información escrita, respectivamente.

La dimensión relaciones interpersonales consideró el trato brindado por los distintos funcionarios que atienden al usuario. Podemos observar que en todos los casos (Tabla 5) sobre el $91,9 \%$ de los usuarios lo califican como muy bueno a bueno.

La dimensión calidad técnica consideró las variables información completa y comprensibilidad de los instructivos para hacer el trámite, claridad de la información entregada y capacidad resolutiva de 
Tabla 3. Porcentaje de usuarios que evaluaron la dimensión accesibilidad

\begin{tabular}{lrr}
\hline Variable & Frecuencia & $\%$ \\
\hline Problemas en ubicación oficinas & & \\
No & 235 & 77,05 \\
$\mathrm{Si}$ & 70 & 22,95 \\
Total & 305 & 100,00 \\
Horario de atención & & \\
Muy bueno & 21 & 9,5 \\
Bueno & 120 & 54,3 \\
Regular & 64 & 29,0 \\
Malo & 14 & 6,3 \\
Muy malo & 2 & 0,9 \\
Total & 221 & 100,0 \\
Tiempo de espera & & \\
Adecuado & 242 & 79,6 \\
Un poco largo & 44 & 14,5 \\
Demasiado largo & 18 & 5,9 \\
Total & 304 & 100,0 \\
Tiempo de tramitación & & \\
Adecuado & 191 & 62,8 \\
Un poco largo & 68 & 22,4 \\
Demasiado largo & 45 & 14,8 \\
Total & 304 & 100,0 \\
Costo de la atención & & \\
Adecuado & 109 & 46,8 \\
Alto & 74 & 31,8 \\
Muy alto & 50 & 100,0 \\
Total & & \\
\hline & & \\
& & \\
\hline
\end{tabular}

Tabla 4. Distribución porcentual de los usuarios que evaluaron la dimensión infraestructura

\begin{tabular}{|c|c|c|c|c|c|c|}
\hline \multirow[t]{3}{*}{ Categorías } & \multicolumn{4}{|c|}{ Característica de las oficinas } & \multirow{2}{*}{\multicolumn{2}{|c|}{ Información escrita }} \\
\hline & \multicolumn{2}{|c|}{ Espacio } & \multicolumn{2}{|c|}{ Limpieza } & & \\
\hline & $\mathbf{N}^{\circ}$ & $\%$ & $\mathbf{N}^{\circ}$ & $\%$ & $\mathrm{~N}^{\circ}$ & $\%$ \\
\hline Muy buena & 10 & 3,32 & 52 & 17,33 & 15 & 5,51 \\
\hline Buena & 161 & 53,49 & 230 & 76,67 & 205 & 75,37 \\
\hline Regular & 111 & 36,88 & 16 & 5,33 & 42 & 15,44 \\
\hline Mala & 17 & 5,65 & 1 & 0,33 & 7 & 2,57 \\
\hline Muy mala & 2 & 0,66 & 1 & 0,33 & 3 & 1,10 \\
\hline Total & 301 & 100 & 300 & 100 & 272 & 100 \\
\hline
\end{tabular}


Tabla 5. Distribución porcentual de los usuarios que evaluaron la dimensión relaciones interpersonales

\begin{tabular}{|c|c|c|c|c|c|c|}
\hline \multirow[t]{3}{*}{ Categorías } & \multicolumn{6}{|c|}{ Calificación del trato } \\
\hline & \multicolumn{2}{|c|}{ Inspector } & \multicolumn{2}{|c|}{ Secretaria } & \multicolumn{2}{|c|}{ Jefe oficina } \\
\hline & $\mathbf{n}^{\circ}$ & $\%$ & $\mathbf{n}^{\circ}$ & $\%$ & $n^{\circ}$ & $\%$ \\
\hline Muy bueno & 136 & 45,95 & 116 & 38,93 & 79 & 48,77 \\
\hline Bueno & 136 & 45,95 & 163 & 54,70 & 77 & 47,53 \\
\hline Regular & 19 & 6,42 & 15 & 5,03 & 6 & 3,70 \\
\hline Malo & 4 & 1,35 & 3 & 1,01 & 0 & 0,00 \\
\hline Muy malo & 1 & 0,34 & 1 & 0,34 & 0 & 0,00 \\
\hline Total & 296 & 100 & 298 & 100 & 162 & 100 \\
\hline
\end{tabular}

Tabla 6. Distribución porcentual de los usuarios que evaluaron la calidad técnica

\begin{tabular}{|c|c|c|}
\hline Variable & Frecuencia & $\%$ \\
\hline \multicolumn{3}{|l|}{ Claridad } \\
\hline Muy clara & 78 & 25,57 \\
\hline Clara & 167 & 54,75 \\
\hline Poco clara & 44 & 14,43 \\
\hline Muy Poco clara & 16 & 5,25 \\
\hline Total & 305 & 100 \\
\hline Información completa & & $\%$ \\
\hline $\mathrm{Si}$ & 155 & 90,12 \\
\hline No & 5 & 2,91 \\
\hline En parte & 12 & 6,98 \\
\hline Total & 172 & 100 \\
\hline Comiprensibilidad & & $\%$ \\
\hline $\mathrm{Si}$ & 126 & 73,26 \\
\hline No & 13 & 7,56 \\
\hline En parte & 33 & 19,19 \\
\hline Total & 172 & 100 \\
\hline Solución a la denuncia & & $\%$ \\
\hline $\mathrm{Si}$ & 13 & 18,6 \\
\hline No & 40 & 57,1 \\
\hline En parte & 17 & 24,3 \\
\hline Total & 70 & 100 \\
\hline
\end{tabular}


las denuncias. De los usuarios que señalaron haber leído los instructivos el $90,12 \%$ opinó que contenían la información necesaria y el 73,26\% que eran comprensibles para ellos (Tabla 6). Por otra parte, el $80,32 \%$ califica como clara o muy clara la información entregada para hacer el trámite. Entre los usuarios que habían presentado denuncias, el resultado muestra que sólo el $18,6 \%$ consideró que el problema denunciado había sido solucionado.

El estudio de asociaciones entre variables independientes entrega algunos resultados de interés. En comparación con el resto, los usuarios que realizaron trámites más complejos y denuncias, evaluaron más mal el tiempo de tramitación ( $\mathrm{p}<$ $0,05)$. Por otro lado, ambos tipos de trámites los realizaron con más frecuencia las mujeres $(p<0,05)$, quienes a su vez tienen un nivel educacional más bajo $(\mathrm{p}<0,05)$ y evaluaron más mal la claridad de la información $(\mathrm{p}<0,1)$ y la comprensibilidad de los instructivos $(\mathrm{p}<0,05)$.

Otro hallazgo indica que existe una asociación estadísticamente significativa ( $p<0,01)$ entre la oficina de atención y la accesibilidad geográfica. El grupo de usuarios atendido en la oficina de LlayLlay es el que evalúa más mal esta variable con un $31,11 \%$ que señala que sí hay problemas con la ubicación. En segundo lugar están los atendidos en la oficina de San Felipe con un 28,79\% de las respuestas en esta misma categoría.

Respecto a la evaluación de la satisfacción global que hicieron los usuarios de todo el proceso de atención, el 73,61\% evaluó con nota $6 \% 7,10$ que es considerado como bueno a muy bueno.

Para conocer las variables que más influyen en la satisfacción global, se estudió la fuerza de asociación y el impacto de algunas variables, a través de la determinación de los Odds Ratios (OR), Intervalos de Confianza (IC 95\%) y Riesgo Atribuible Porcentual (RAP\%). Los resultados de las variables sociodemográficas, oficina de atención y tipo de trámite, sobre la satisfacción global, muestran una asociación estadísticamente significativa entre esta variable y el tipo de trámite (Tabla 7). Esto indica que los usuarios que realizaron trámites más complejos y denuncias evaluaron más mal la atención con un $37,40 \%$ de respuestas con satisfacción global calificada con nota 1-5, en comparación con los que hicieron trámites menos complejos entre los cuales sólo el 18,18\% la calificó en esta categoría.

Respeto a las variables que medían las diferentes dimensiones en la Tabla 8 se observa que aquellas que más impactan la evaluación de la satisfacción global de los usuarios son la claridad de la información $(\mathrm{RAP} \%=81 \%$ ), el tiempo de tramitación de las solicitudes (RAP\% $=79 \%$ ) y el trato brindado por los inspectores (RAP\% $=75,9 \%$ ). Las variables que presentan menor impacto, dado los más bajos RAP\%, son las relacionadas con la infraestructura, accesibilidad geográfica, horarios y costo de la atención.

Las preguntas abiertas de la encuesta permitieron validar que los aspectos prioritarios de la atención son la accesibilidad en términos de la accesibilidad organizacional y de la oportunidad de la atención, y que las relaciones humanas es la dimensión mejor evaluada por los usuarios. Por otra parte, no surgieron dimensiones diferentes a las consideradas en las preguntas cerradas.

Tabla 7. Asociación e impacto de las variables sociodemográficas y tipo de trámite en la satisfacción global de los usuarios

\begin{tabular}{lccccc} 
Variable & $\begin{array}{c}\text { \% respuestas } \\
\text { negativas entre } \\
\text { expuestos }\end{array}$ & OR & IC 95\% & p & RAP\% \\
\hline $\begin{array}{l}\text { Edad (53-77 años) } \\
\text { Sexo (mujeres) }\end{array}$ & 31,08 & 1,36 & $0,77-2,41$ & 0,2927 & 22,58 \\
$\begin{array}{l}\text { Nivel de educación } \\
\text { (analfabeto-básico) }\end{array}$ & 22,95 & 1,67 & $0,99-2,8$ & 0,0525 & 32,25 \\
Tipo de trámite* & 37,40 & 0,8 & $0,42-1,53$ & 0,4967 & 18,18 \\
\hline
\end{tabular}

* Trámites mal evaluados: Muy complejo, complejo y denuncias. 
Tabla 8. Asociación e impacto de las variables que miden las diferentes dimensiones de la atención

\begin{tabular}{|c|c|c|c|c|c|c|}
\hline \multirow[t]{2}{*}{ Variable } & \multicolumn{2}{|c|}{ Resp. Negativas } & \multirow[t]{2}{*}{ OR } & \multirow[t]{2}{*}{ IC $95 \%$} & \multirow[t]{2}{*}{$\mathbf{p}$} & \multirow[t]{2}{*}{ RAP\% } \\
\hline & $\mathbf{N}^{0}$ & $\begin{array}{l}\% \text { entre * } \\
\text { expuestos }\end{array}$ & & & & \\
\hline Problemas ubicación oficinas & 70 & 30,00 & 1,27 & $0,71-2,21$ & 0,4139 & 16,66 \\
\hline Horarios de atención & 80 & 37,50 & 3,08 & $1,64-5,79$ & 0,0004 & 57,89 \\
\hline Tiempo espera para ser atendido & 62 & 64,52 & 9,18 & $4,95-18,02$ & 0,0000 & 73,85 \\
\hline Tiempo respuesta a la solicitud & 113 & 50,44 & 7,40 & $4,21-18,11$ & 0,0000 & 76,00 \\
\hline Información completa en instructivos & 10 & 58,82 & 5,95 & $2,15-16,43$ & 0,0003 & 67,79 \\
\hline Instructivos comprensibles & 21 & 45,65 & 4,73 & $2,23-10,04$ & 0,0000 & 67,39 \\
\hline Claridad de la información & 60 & 76,67 & 20,39 & $10,19-40,76$ & 0,0000 & 81,81 \\
\hline Calificación conocimientos $* *$ & 33 & 89,19 & - & - & . & - \\
\hline Limpieza y orden & 18 & 61,11 & 5,14 & $1,97-13,38$ & 0,0004 & 62,29 \\
\hline Espacio físico & 130 & 36,15 & 2,56 & $1,51-4,23$ & 0,0004 & 50,00 \\
\hline Información escrita & 52 & 48,00 & 3,60 & $1,91-6,77$ & 0,0000 & 58,33 \\
\hline Trato secretaria & 19 & 63,16 & 5,64 & $2,19-14,49$ & 0,0001 & 63,49 \\
\hline Trato inspector & 20 & 83,83 & 19,73 & $6,75-53,30$ & 0,0000 & 75,90 \\
\hline Trato jefe oficina $* *$ & 6 & no & - & - & - & - \\
\hline $\begin{array}{l}\mathrm{N}^{\circ} \text { de veces que acudió a hacer el } \\
\text { trámite }\end{array}$ & 140 & 35,71 & 2,46 & $1,46-4,16$ & 0,0007 & 50 \\
\hline Costo de la atención & 123 & 27,42 & 2,19 & $1,14-4,22$ & 0,0181 & 44,44 \\
\hline
\end{tabular}

* Porcentaje que evaluaron mal la variable dependiente y que se mostraron insatisfechos con la satisfacción global.

** No se pudo calcular los OR, pero presentaron un p value de 0,000 y 0,001 , respectivamente

\section{DISCUSIÓN}

La satisfacción del usuario es un resultado deseado en la atención y se emplea cada vez con más frecuencia para evaluar la calidad de la atención. La expresión de la satisfacción o no satisfacción es el juicio del usuario sobre la calidad de la atención en todos sus aspectos, pero-generalmente-referido a un proceso interpersonal ${ }^{12+13}$. Su estudio permite identificar las brechas existentes entre las expectativas de los usuarios y la calidad del servicio prestado y arroja información valiosa que ayuda a orientar la toma de decisiones para lograr brindar un servicio más eficiente y centrado en las necesidades de los usuariost.

El estudio considera dos aspectos que son significativos. En primer lugar, es uno de los pocos en el país que evalúan la modalidad de atención que se presta en salud ambiental y es el primero en el DPA del Servicio de Salud Aconcagua. En segundo lugar, fue concebido dentro de una línea de tra- bajo del Servicio de Salud Aconcagua, que intenta fortalecer la aplicación de las políticas de modernización del Estado, incorporando el concepto de la calidad de los servicios prestados.

Del estudio se desprende que las principales brechas del sistema son la accesibilidad organizacional y la capacidad resolutiva de algunas de las prestaciones que se brindan. Respecto a la primera y específicamente al tiempo de tramitación de las solicitudes, más de un tercio de los usuarios encuestados $(37,2 \%)$, es decir 113 personas, muestran algún grado de insatisfacción con esta variable. Por otra parte, si se analiza los RAP\%, se observa que intervenir sobre ella permitiría disminuir la insatisfacción en un $76 \%$. Un elemento orientador es que la insatisfacción con esta variable guarda relación con la complejidad del trámite, ya que si bien los RAP\% no son los más altos para los trámites complejos y las denuncias, éstos concentran el $51,9 \%$ del total de usuarios que asignan la calificación más baja a la satisfacción global y 
entre sus grupos representan el mayor porcentaje de respuestas insatisfactorias, lo que indicaría que son variables relevantes sobre las que intervenir.

En cuanto a la capacidad resolutiva, sólo un $18,6 \%$ se manifiesta totalmente conforme con la respuesta a su denuncia, resultado que es concordante con la mala evaluación realizada por los denunciantes en un estudio chileno ${ }^{14}$. Este aspecto es relevante, si se toma en cuenta que este hecho puede conducir a que los usuarios no demanden atención o comenten esta insatisfacción a otras personas ${ }^{15}$, contribuyendo de esta forma a disminuir la credibilidad y la adhesión de los ciudadanos a las instituciones públicas. Mejorar esta situación implica fortalecer la capacidad fiscalizadora de los Servicios de Salud, dotándolos de los recursos físicos y humanos que les permitan responder oportuna y eficazmente a las demandas de la comunidad.

Llama la atención en el estudio, el alto grado de satisfacción global observado en los usuarios. En general, los estudios muestran que, aunque los usuarios evalúen mal determinadas variables de la atención brindada $8,16,17$, al evaluar la atención global tienden a calificarla en términos más bien positivos. Este hallazgo, junto a la buena evaluación que hicieron los usuarios al trato brindado por los funcionarios y al alto impacto de esta variable en la satisfacción global, conducen a pensar que el usuario al momento de emitir esta opinión pondera los distintos aspectos de la atención, asignándole más valor a la calidad del trato recibido, que a las dificultades de acceso al sistema. Por otra parte, podría reflejar una característica distintiva en los funcionarios del DPA o estar influenciado porque la mayor parte de los trámites realizados correspondieron a aquéllos definidos como poco complejos. Tampoco se puede descartar que está situación exprese un sesgo asociado al temor a represalias por parte de los usuarios, considerando que la mayor parte de ellos son, y continuarán siendo a futuro, fiscalizados por los mismos funcionarios que los atienden. Otra explicación es que esta situación esté influenciada por una percepción negativa previa, fundada o infundada, tomando en cuenta que nuestra cultura tiende a no comprender el rol fiscalizador de las Instituciones y que, debido a ello, los usuarios hayan tenido menores expectativas respecto al trato que recibirían.

Por otra parte, se observaron limitaciones en la accesibilidad geográfica para los usuarios de las oficinas de San Felipe y Llay-Llay, las que podrían ser explicadas porque efectivamente en estos casos existe mayor distancia desde los lugares de procedencia de los usuarios, menor disponibilidad de movilización hasta las oficinas y a que éstas se ubican más lejos de los lugares donde se concentran las instituciones donde los usuarios acuden a realizar más frecuentemente trámites. No es posible saber con exactitud cuántos de los usuarios no acuden en busca de atención debido a este factor, pero no se puede descartar que ello influya, fundamentalmente en aquellas personas que viven en sectores rurales, en quienes tienen menor nivel socioeconómico y en aquellos que deseen hacer una denuncia, ya que para éstos últimos no existe una obligación legal de hacer el trámite.

De acuerdo a lo señalado por el Ministerio de Salud ${ }^{10}$, la edad, el sexo y el nivel educacional son también variables que influyen en el grado de satisfacción de los usuarios. Concordante con ello, en esta investigación se consideró la inclusión de estas variables. Si bien la proporción de mujeres y hombres que acuden en busca de atención es similar, las mujeres evalúan más mal la atención global que los hombres, lo que podría deberse a que ellas realizan un mayor porcentaje de trámites complejos y más denuncias en comparación con los hombres. Estos trámites son los que fueron más mal evalua dos por los usuarios a través de la satisfacción global. Puede estar influyendo que las mujeres usuarias tienen un menor nivel educacional, lo que les podría dificultar la comprensión y, por ende, el cumplimiento de las exigencias de los trámites más complejos, incidiendo de este modo en una mala evaluación de la claridad de la información.

En relación al grado de satisfacción con las variables que evalúan las características de la infraestructura, el espacio físico es mal evaluado consistentemente en todas las oficinas. Este resultado no es coincidente con la evaluación de esta dimensión que hacen los usuarios atendidos en la atención primaria u hospitalaria, quienes -en general- la evalúan mejor. No es posible saber si esta diferencia en la opinión de los usuarios respecto a diferentes tipos de infraestructura, se explique por diferencias efectivas en esta variable, o bien a factores como diferencias en el nivel educacional de los usuarios de uno u otro tipo de servicios.

Es interesante destacar que, si bien es cierto, variables como el espacio físico, los horarios y el costo 
de la atención, mostraron la más mala evaluación, ellas presentan un menor impacto en la satisfacción global y otras, como el trato y la claridad de la información, que fueron mejor evaluadas, se muestren más relacionadas con una mala satisfacción global, hallazgo que es similar con los de un estudio realizado en hospitales chilenos ${ }^{18}$. Esto es importante porque entrega información que permite orientar los esfuerzos a abordar las situaciones que realmente conducirán a mejorar la satisfacción usuaria.

En el contexto de la creciente preocupación de la opinión pública por el tema de la probidad, llama la atención que en las preguntas abiertas no se manifestaran dimensiones relacionadas con la transparencia o probidad de los funcionarios, lo que podría estar indicando una percepción favorable del usuario, a juzgar por el hecho que no surgió espontáneamente en su discurso como un tema relevante. Por otra parte, nuevamente no es descartable que se hayan mostrado renuentes a expresarlo por temor a represalias.

El estudio permitió identificar las variables con menor nivel de satisfacción, aquellas que más impactan la satisfacción global de los usuarios y formular algunas sugerencias para mejorar aquellos aspectos deficientes y potenciar aquellos mejor evaluados. Esta información constituye un insumo para los profesionales, técnicos y administrativos que se desempeñan en el DPA $y$, eventualmente para otros Servicios de Salud, especialmente para quienes ocupan posiciones en las cuales deben tomar decisiones, que les permite justificar la implementación de acciones específicas para mejorar la atención de los usuarios.

\section{AGRADECIMIENTOS}

A la Representación de la Organización Panamericana de la Salud en Chile, por el fịnanciamiento brindado para la ejecución de la presente investigación, mediante una subvención para tesis de Magíster en Salud Pública.

A los Directivos del Servicio de Salud Aconcagua, quienes impulsaron el desarrollo de esta investigación y brindaron todo el apoyo necesario para la ejecución.

\section{REFERENCIAS}

1. MINISTERIO SECRETARIA GENERAL DE LA PRESIDENCIA. La Calidad al Scrvicio de los ciu- dadanos. Bases y Guía. Premio Nacional a la Calidad de los Servicios Públicos 2001.

2. - VARO J. Gestión estratégica de la calidad en los servicios sanitarios. Madrid: Editorial Díaz de Santos, 1993. Citado por Roldán P, Vargas C, Giraldo C, Valencia G, García C, Salamanca Evaluación de la calidad en la atención en salud. Un estudio de caso. Colombia Médica 2001; 32: 14-8.

3. - CASAS A. Gobernabilidad, salud y reforma: Hacia el desarrollo humano y la salud con equidad: En Taller sobre Gobermabilidad y Gobemancia en Salud; 1998 Diciembre 8 y 9; Washington DC, EEUU.

4. - HAYES B. Cómo medir la satisfacción del cliente. Desarrollo y utilización de cuestionarios. 2 da ed. Barcelona: Ediciones Gestión 20/00, S.A. 1999.

5. - MINISTERIO DE SALUD. Objetivos sanitarios y modelo de salud para la década 2000-2010. Documento Oficial. Enero 2002.

6.- ORDONEZ G. Salud ambiental: Conceptos y actividades. Organización Panamericana de la Salud. Rev Panam Salud Pública 2000, 7(3): 137-47.

7. - URRUTIA M. Recursos humanos para una gestión optimizala en el Departamento Programas Sobre el Ambiente del Servicio de Salud de BíoBío. Tesis (Magister en Salud Pública). Santiago: Universidad de Chile, 1992.

8. - MINISTERIO DE SALUD y Corporación Municipal de la Florida. Evaluación de la Calidad de la Atención en la Comuna de la Florida: El punto de vista de los usuarios, 1994.

9. - MINISTERIO DE SALUD, División de Salud de las Personas, Departamento de Atención Primaria. Evaluación de la Atención Primaria. Ira ed. Santiago: Ediciones Paulinas Ltda., 1997. citados por S.R. Steiberg y W. J. Krowinski en Mesuring and Managing Patient Satisfaction.

10. MINISTERIO DE SALUD, Programa de Mejoramiento de la Calidad. Marco conceptual en busca de la calidad de la atención. Módulo $\mathrm{N}^{\circ} 2,1997$. Pág. 3.

11. -DE LEÓN O, ARTEAGA O, SCHIATTINO I. Medición del nivel de satisfacción de usuarios que asisten al Centro de Salud de Acatenango, Guatemala. Rev Chil Salud Pública 2003 ; Vol 7 (2):80-7.

12. GAZITÚA H R. Control continuo de calidad $y$ costo efectividad de problemas médicos comunes. Boletín Escuela de Medicina. Pontificia Universidad Católica de Chile 1995, 24: 174-8.

13. -ARANAZ J M, MIRA J, MARTÍNEZ L. La valoración de los resultados generales de la actividad asistencial en los servicios de cirugía. Revista de Cirugía Española 1999, 66: 433-44.

14.- CONAMA. Evaluación de eficiencia y efectividad de normas ambientales vigentes generadas por Conama. Informe final. Julio 2002. Pag 8-13. http:/ /www.conama.cl/portal/1255/articles 26351_documento_1.pdf.

15.- WHO. Center for Communication Programs. Populations Report. Family planning programs: 
improving quality. population information program, USA. Volumen XXVI, Número 3 Noviembre de 1998. Serie J, Número 47. http://www.jhucep.org/ $\mathrm{pr} / \mathrm{j} 47 \mathrm{edsum} . \mathrm{stm}$.

16.- LINN M, LINN B, STEIN S. Satisfaction with ambulatory care and compliance in older patients. Med. Care 1982; 20(6): 606-14. Citado por Wall V. Atención de Salud y satisfacción de usuarios adultos en los distintos subsistemas de salud en el gran Santiago. Tesis (Magister en Salud Pública). Santiago: Universidad de Chile, 1994.
17. -SERVICIO DE SALUD METROPOLITANO DEL AMBIENTE. Comunicación personal Sr. Ronnie Ramírez, Jefe Subdepartamento Evaluación de Proyectos y Control de Gestión, Servicio de Salud Metropolitano del Ambiente, Santiago, Región Metropolitana. Entrevista realizada en noviembre del año 2001.

18. -ALVARADO R, VERA A. Evaluación de la Satisfacción de usuarios de hospitales del sistema nacional de servicios de salud en Chile. Rev Chil SaJud Pública 2001; Vol 5 (2-3): 81-9.

Usted puede comentar éste y otros artículos publicados en la Revista Chilena de Salud Pública, enviando un correo electrónico a revistasp@med.uchile.cl 